\title{
PULMONARY ARTERY GROWTH AFTER BIDIRECTIONAL CAVOPULMONARY SHUNT: IS THERE A CAUSE FOR CONCERN?
}

V. Mohan Reddy, MD

Doff B. McElhinney, $\mathrm{MS}^{\mathrm{a}}$

Phillip Moore, $\mathrm{MD}^{\mathrm{b}}$

Edwin Petrossian, $\mathrm{MD}^{\mathrm{a}}$

Frank L. Hanley, MD
Objective: Our objective was to analyze changes in pulmonary artery size after bidirectional cavopulmonary shunt. Methods: All 47 patients who underwent bidirectional cavopulmonary shunt between March 1990 and May 1995 who had preoperative and postoperative angiograms available for review were selected for study. This included 24 patients who had also undergone a modified Fontan operation. Clinical records were reviewed retrospectively and cross-sectional follow-up was obtained by direct physician contact. Angiograms were reveiwed, and the right and left pulmonary artery diameters were each measured at two locations: immediately distal to their origin and at the narrowest point. In addition, the lower lobe pulmonary artery branch was measured just distal to its origin. Cross-sectional areas (left, right, and total) at each point of measurement were indexed to body surface area. Angiographic and hemodynamic data were analyzed. Results: Changes in the various measures of pulmonary artery size after bidirectional cavopulmonary shunt varied considerably. On average the absolute diameters increased for all measures, but the increase in diameter was significant only for the lower lobe arteries. All pulmonary artery indices decreased on average, but these changes did not approach significance. Patients who underwent pulmonary artery augmentation at the time of bidirectional cavopulmonary shunt had significantly smaller pulmonary artery indices before pulmonary artery augmentation, relative to those who did not undergo pulmonary artery repair, and significantly greater changes (possibly to a large extent owing to pulmonary artery repair) in the right and left pulmonary artery index after bidirectionl cavopulmonary shunt. Lower lobe indices did not differ preoperatively or exhibit different degrees of change in size between patients who did and did not undergo pulmonary artery repair. One patient died after Fontan completion (pulmonary artery index: 305 $\mathrm{mm}^{2} / \mathrm{m}^{2}$ ), and none of the factors analyzed correlated with Fontan outcomes. Conclusions: A more appropriate measure of pulmonary artery growth is the indexed cross-sectional area of the lower lobe branch of the right and left pulmonary arteries, which is less likely to be altered surgically with systemicpulmonary shunts, pulmonary artery repair, and the bidirectional cavopulmonary anastomosis itself. Pulmonary artery indices, including the lower lobe index, do not change significantly after bidirectional cavopulmonary shunt during medium-term follow-up and do not influence the Fontan outcome. (J Thorac Cardiovasc Surg 1996;112:1180-92)
$P_{t}$ ulmonary artery (PA) size is generally believed to influence the outcomes of the modified Fontan operation, although various reports have presented conflicting data regarding the nature of this

From the Divisions of Cardiothoracic Surgery a and Pediatric Cardiology, ${ }^{\mathrm{b}}$ University of California, San Francisco, San Francisco, Calif.

Read at the Seventy-sixth Annual Meeting of The American Association for Thoracic Surgery, San Diego, Calif., April 28-May 1, 1996. relationship. ${ }^{1-6}$ Increasingly, a bidirectional cavopulmonary shunt (BCPS) is being used as interim palliation before a modified Fontan operation for

Received for publication May 6, 1996; revisions requested June 25, 1996; revisions received August 7, 1996; accepted for publication August 7, 1996.

Address for reprints: V. Mohan Reddy, MD, 505 Parnassus Ave., M593, San Francisco, CA 94143-0118.

Copyright (C) 1996 by Mosby-Year Book, Inc.

$0022-5223 / 96 \$ 5.00+0 \quad \mathbf{1 2 / 6 / 7 7 1 4 0}$ 
patients with single ventricle. ${ }^{7-10}$ Thus, the effect of BCPS on PA growth may influence the success of subsequent Fontan completion. Because pulmonary blood flow, which is thought to be a stimulus for PA growth, ${ }^{11}$ is usually decreased in BCPS (depending on the presence and volume of an auxiliary source of flow), leaving patients with a BCPS for extended periods may result in suboptimal PA growth. This may have implications for the timing of BCPS and subsequent Fontan completion. Reported studies of PA sizes after BCPS have found that indexed PA sizes decrease after BCPS, although absolute changes in PA size have varied among studies. ${ }^{12-14}$ To address the questions of how BCPS influences PA growth, what factors may be associated with better PA growth in the presence of BCPS, and how important these factors ultimately are with respect to outcomes after the modified Fontan operation, we analyzed pre-BPCS and post-BPCS angiograms in 47 patients, 24 of whom have undergone Fontan completion.

\section{Patients and methods}

Patients and operative procedures. Between March 1990 and May 1995, 105 patients underwent BCPS. Of these, 54 have undergone both pre-BCPS and post-BCPS catheterization. Preoperative angiograms were unavailable for five patients, and post-BCPS films were unavailable for an additional two patients. The remaining 47 patients comprise the study group for the present report. Patient diagnoses are summarized in Table I. BCPS was the first surgical procedure (primary BCPS) performed in five patients; the remaining 42 patients had undergone one $(n=30)$ or more $(n=12)$ previous palliative procedures, including a systemic-PA shunt in 31 patients (a modified or classic left-sided Blalock-Taussig shunt in seven patients, a right-sided modified Blalock-Taussig shunt in 17 patients, a central shunt in six patients, and bilateral Blalock-Taussig shunts in one patient).

Median age at operation was 1.3 years (range 1 month to 43 years). Unilateral right BCPS was performed in 35 patients, whereas all 12 patients with bilateral superior venae cavae underwent bilateral BCPS. Twenty-one patients underwent PA augmentation procedures. A source of pulmonary blood flow in addition to the BCPS was placed $(n=4)$ or allowed to remain (from the native anatomy or a previous procedure; $n=27$ ) in 31 patients $(66 \%)$, as either antegrade flow through a banded or stenotic main PA $(n=10)$ or a systemic-PA shunt $(n=$ 21).

Measurement of PA size and hemodynamics. PreBCPS and post-BCPS angiograms were reviewed retrospectively. Right and left branch PAs were measured just distal (about $5 \mathrm{~mm}$ ) to their origin and at the narrowest point in the right and left branch $P A$, which was almost invariably medial to the BCPS. The post-BCPS narrowest point was not necessarily the same as the pre-BCPS site.
Table I. Diagnoses

\begin{tabular}{lc}
\hline \multicolumn{1}{c}{ Diagnosis } & No. of patients \\
\hline DILV & 9 \\
Hypoplastic left heart syndrome & 7 \\
Tricuspid atresia & 7 \\
Heterotaxy & 5 \\
Pulmonary atresia with IVS & 5 \\
Mitral atresia & 3 \\
Ebstein's anomaly (no DILV) & 1 \\
Other complex single ventricle & 10 \\
\hline
\end{tabular}

DILV, Double-inlet left ventricle; $I V S$, intact ventricular septum.

When the narrowest point diameter was less than $75 \%$ of the diameter of the branch PAs, the branch PA in question was defined as having a significant stenosis. Because many patients underwent PA augmentation at the time of BCPS, it was hypothesized that the PA index ${ }^{3}$ might prove to be a misleading indicator of actual PA growth. Therefore, in addition to measuring the left and right PAs, the diameters of the right and left lower lobe PAs were measured immediately distal to their origins. All measurements were made twice, and the average of the two values was recorded for analysis. Measurements were corrected for magnification by relating measured diameters to the measured catheter diameter, then calibrating the ratio according to the known catheter size.

Indices were determined by calculating cross-sectional area (square millimeters, with the assumption of circular cross-sectional area at the points measured) and dividing this by body surface area (square meters), giving units of square millimeters per square meter. Indices were calculated for all diameters measured: proximal right and left branch PAs, with the sum of these two being called "total" PA index; lower lobe artery immediately distal to its origin on each side (right and left), with the sum of these two being total lower lobe index; and at the narrowest point in each branch PA (right and left), with the sum of these two being the narrowest point index. In addition, descending aortic diameter was measured immediately proximal to the diaphragm, and cross-sectional area was indexed to body surface area. Differences between pre-BCPS and post-BCPS PA diameters and indices were determined by simple subtraction. Whenever possible, these differences are referred to in the text as "size changes" or "diameter/ index changes," rather than as "growth," which is an interpretive assessment.

In addition, angiograms were analyzed for lobar perfusion defects and for the presence of systemic-PA collateral arteries. Systemic-PA collaterals were defined as vessels clearly observed to originate from the arterial system, on aortography, and to opacify the pulmonary arteries, veins, or both.

Hemodynamic data obtained at pre-BCPS and postBCPS cardiac catheterization were noted. Pre-BCPS pulmonary blood flow was estimated by the Fick method in patients with a single source of pulmonary blood flow. Pulmonary vascular resistance was calculated by dividing the transpulmonary pressure gradient by pulmonary blood flow. Of note, two thirds of our patients had a source of pulmonary blood flow in addition to the BCPS, and hence 
Table II. Pre-BCPS PA sizes and indices

\begin{tabular}{|c|c|c|c|c|c|c|c|c|c|c|c|c|}
\hline & \multicolumn{5}{|c|}{ Right $P A$} & \multicolumn{5}{|c|}{ Left $P A$} & \multicolumn{2}{|c|}{ Totalt } \\
\hline & $\begin{array}{c}\text { Origin } \\
\text { diameter* }\end{array}$ & $\begin{array}{c}\text { NP } \\
\text { diameter* }\end{array}$ & $\begin{array}{c}L L \\
\text { diameter* }\end{array}$ & $\begin{array}{l}\text { Origin } \\
\text { indext }\end{array}$ & $\begin{array}{c}L L \\
\text { indext }\end{array}$ & $\begin{array}{c}\text { Origin } \\
\text { diameter* }\end{array}$ & $\begin{array}{c}N P \\
\text { diameter* }\end{array}$ & $\begin{array}{c}L L \\
\text { diameter* }\end{array}$ & $\begin{array}{l}\text { Origin } \\
\text { indext }\end{array}$ & $\begin{array}{c}L L \\
\text { indexf }\end{array}$ & $\begin{array}{c}P A \\
\text { indext }\end{array}$ & $\underset{\text { indext }}{L L}$ \\
\hline All patients & $8.7 \pm 5.4$ & $7.2 \pm 5.3$ & $7.4 \pm 3.5$ & $125 \pm 71$ & $99 \pm 50$ & $9.0 \pm 5.0$ & $7.1 \pm 3.7$ & $7.2 \pm 3.4$ & $135 \pm 77$ & $90 \pm 44$ & $256 \pm 180$ & $183 \pm 76$ \\
\hline \multicolumn{13}{|l|}{$\begin{array}{c}\text { Additional } \\
\text { PBF }\end{array}$} \\
\hline Yes & $11.1 \pm 5.3$ & $9.1 \pm 6.6$ & $7.9 \pm 3.9$ & $167 \pm 111$ & $96 \pm 48$ & $10.0 \pm 5.0$ & $7.9 \pm 3.7$ & $8.2 \pm 3.7$ & $147 \pm 61$ & $105 \pm 45$ & $314 \pm 149$ & $195 \pm 77$ \\
\hline No & $5.3 \pm 2.5$ & $4.2 \pm 1.8$ & $6.4 \pm 1.9$ & $77 \pm 60$ & $103 \pm 55$ & $7.0 \div 4.8$ & $5.5 \pm 3.0$ & $5.5 \pm 1.8$ & $111 \pm 99$ & $63 \pm 26$ & $182 \pm 116$ & $180 \pm 67$ \\
\hline \multicolumn{13}{|l|}{$\begin{array}{l}\text { Bilateral } \\
\text { BCPS }\end{array}$} \\
\hline Yes & $8.3 \pm 2.9$ & $7.4 \pm 6.0$ & $7.7 \pm 3.6$ & $116 \pm 73$ & $99 \pm 46$ & $7.7 \pm 2.8$ & $6.0 \pm 2.6$ & $6.3 \pm 2.2$ & $132 \pm 60$ & $86 \pm 36$ & $281 \pm 107$ & $183 \pm 74$ \\
\hline No & $8.9 \pm 6.1$ & $6.7 \pm 3.1$ & $6.6 \pm 3.1$ & $149 \pm 60$ & $96 \pm 60$ & $9.5 \pm 5.6$ & $7.4 \pm 3.9$ & $7.6 \pm 3.7$ & $136 \pm 84$ & $91 \pm 47$ & $246 \pm 132$ & $183 \pm 78$ \\
\hline \multicolumn{13}{|l|}{ PA repair } \\
\hline Yes & $9.4 \pm 6.4$ & $7.4 \pm 6.1$ & $8.1 \pm 3.6$ & $109 \pm 66$ & $102 \pm 55$ & $8.7 \pm 4.4$ & $6.2 \pm 3.6$ & $7.7 \pm 3.4$ & $116 \pm 58$ & $87 \pm 42$ & $225 \pm 108$ & $189 \pm 68$ \\
\hline No & $8.2 \pm 4.6$ & $7.0 \pm 4.6$ & $6.8 \pm 3.3$ & $137 \pm 73$ & $96 \pm 47$ & $9.2 \pm 5.4$ & $7.7 \pm 3.7$ & $6.9 \pm 3.4$ & $148 \pm 87$ & $93 \pm 46$ & $278 \pm 134$ & $178 \pm 83$ \\
\hline \multicolumn{13}{|l|}{$\begin{array}{r}\text { Status post } \\
\text { shunt }\end{array}$} \\
\hline Yes & $8.9 \pm 6.2$ & $7.5 \pm 6.2$ & $7.7 \pm 3.8$ & $116 \pm 73$ & $101 \pm 53$ & $9.0 \pm 5.0$ & $6.6 \pm 3.7$ & $7.4 \pm 3.6$ & $128 \pm 65$ & $89 \pm 43$ & $242 \pm 128$ & $185 \pm 71$ \\
\hline Left BTS & $13.6 \pm 7.0$ & $12.4 \pm 7.3$ & $10.0 \pm 4.5$ & $169 \pm 54$ & $126 \pm 48$ & $11.2 \pm 4.3$ & $6.9 \pm 3.5$ & $9.7 \pm 3.5$ & $155 \pm 5.3$ & $116 \pm 37$ & $324 \pm 82$ & $250 \pm 71$ \\
\hline Right BTS & $7.2 \pm 5.8$ & $6.3 \pm 5.4$ & $7.5 \pm 3.9$ & $96 \pm 77$ & $103 \pm 60$ & $8.7 \pm 5.7$ & $6.4 \pm 4.2$ & $6.7 \pm 3.9$ & $123 \pm 70$ & $78 \pm 44$ & $215 \pm 141$ & $170 \pm 70$ \\
\hline No & $8.3 \pm 2.8$ & $6.4 \pm 1.9$ & $6.6 \pm 2.3$ & $146 \pm 63$ & $92 \pm 42$ & $9.0 \pm 5.2$ & $8.1 \pm 3.6$ & $6.9 \pm 2.8$ & $149 \pm 100$ & $92 \pm 48$ & $284 \pm 118$ & $178 \pm 88$ \\
\hline
\end{tabular}

Data are presented as mean \pm standard deviation. BCPS, Bidirectional cavopulmonary shunt; BTS, Blalock-Taussig shunt (classic or modified); LL, lower lobe; $N P$, narrowest point; $P A$, pulmonary artery; $P B F$, pulmonary blood flow.

*Diameters are given in millimeters.

†Index units are square millimeters per square meter.

$¥$ Total refers to the sum total of left and right indices, averaged.

pulmonary blood flow could not be calculated accurately. Pre-BCPS and post-BCPS oxygen saturations were also noted from the catheterization records.

Data analysis. Preoperative and perioperative data were collected on retrospective review of patient clinical records. Cross-sectional follow-up was carried out by contact with the referring physician and was completed by February 1996. SPSS for Windows version 7.0 (SPPS Inc., Chicago, Ill.) was used to perform statistical calculations. Data are expressed as median and range, mean \pm standard deviation, or mean and $95 \%$ confidence intervals (CI). Paired two-tailed $t$ test analysis was used to assess the significance of within-patient changes in catheterization and angiography data between pre-BCPS and postBCPS catheterization for each patient. Fisher's exact test and unpaired $t$ test were used to compare mean values between groups. General factorial analysis of variance was used for multifactorial comparison of means. The significance of correlations between continuous variables was determined by linear regression analysis. Variables analyzed are listed in the appendix. PA sizes were also compared against previously published normative values. ${ }^{15}$ All $p$ values of less than or equal to 0.05 were considered significant.

\section{Results}

Pre-BCPS PA sizes and catheterization data. Pre-BCPS catheterization and angiography were performed between 1 day and 10 months before the operation (median 21 days). Pre-BCPS PA diameters and indices are presented in Table II. Both left and right PA diameters, plotted against body surface area, were comparable with reported angiographic measurements in individuals with no known cardiovascular or pulmonary disease (Fig. 1) ${ }^{15}$ Twenty patients were observed to have significant right branch PA stenosis (narrowest point diameter $<75 \%$ of bifurcation diameter), and 13 patients had significant left PA stenosis. No significant differences in PA size or hemodynamics were found between patients with and without preoperative shunts. Pre-BCPS hemodynamic and oximetric data are summarized in Table III.

Post-BCPS PA sizes and catheterization data. The median duration from BCPS to post-BCPS angiography was 16 months (range 1 to 47 months). Post-BCPS PA diameters and indices and pre-BCPS to post-BCPS changes are summarized in Table IV. Descending aortic diameters increased significantly $(p<0.0001)$, but the indexed cross-sectional area did not change $(p=0.68)$.

$P A$ diameters. As was the case before the operation, post-BCPS left and right PA diameters were almost identical to normal values (Fig. 1). ${ }^{15}$ Changes in PA and lower lobe artery diameters varied considerably. Both mean left and right lower lobe artery diameters increased significantly (left, $p=0.011$; right, $p=0.034$ ), whereas the changes in left and right PA diameters were insignificant (left, $p=0.96$; right, $p=0.092)$. Changes in right $(p=0.04)$ and 


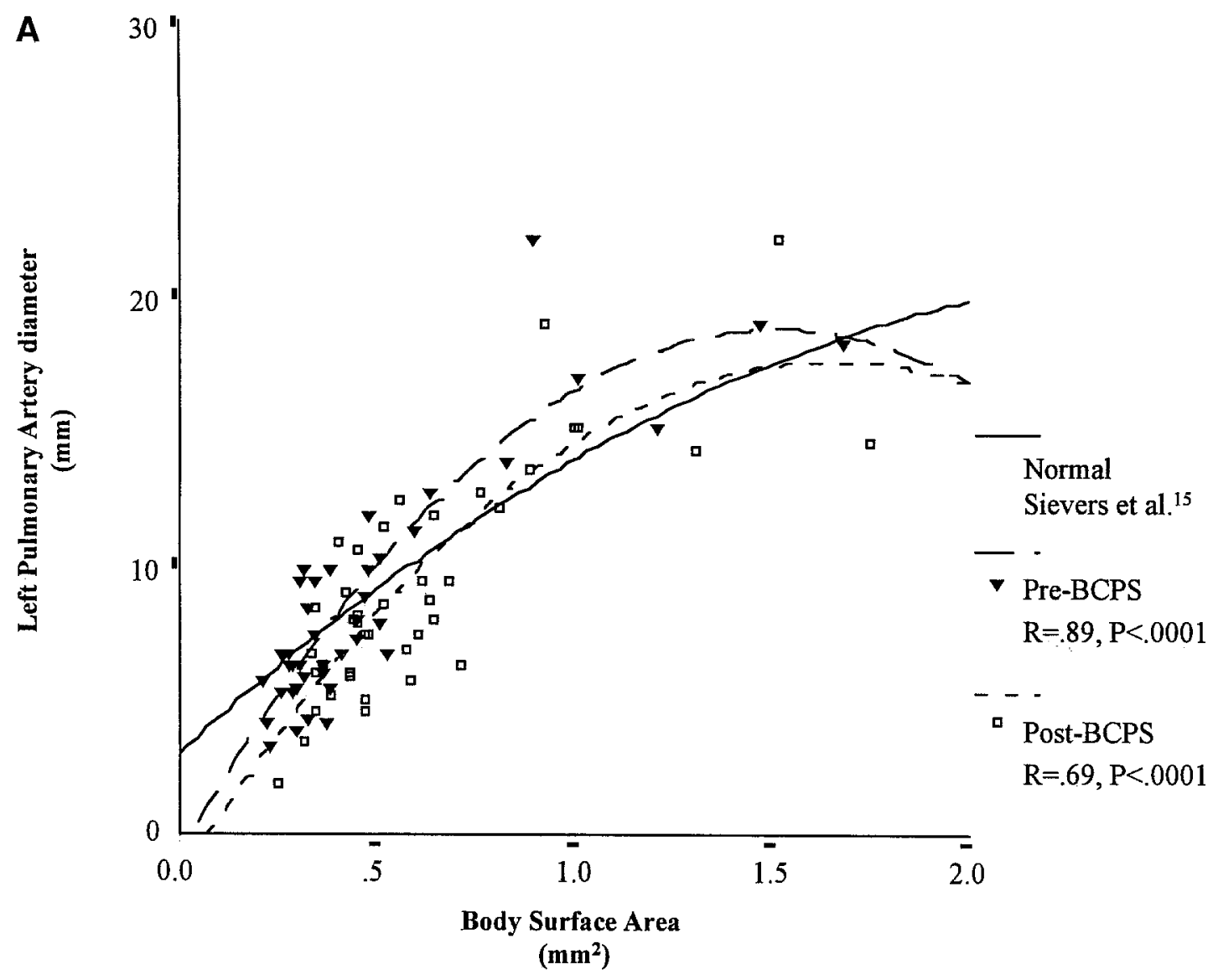

Fig. 1A. Pre-BCPS and post-BCPS left PA diameters (bifurcation) plotted against body surface area and compared with normal values published by Sievers and associates. ${ }^{15}$

left $(p=0.04)$ PA diameter were significantly greater in patients who underwent $P A$ augmentation at BCPS than in those who did not. However, there was no significant difference in lower lobe artery changes between patients who did and did not undergo PA augmentation. Twelve patients had significant right branch PA stenosis and 11 had significant left branch PA stenosis.

$P A$ indices. The overall mean total PA index and total lower lobe index decreased after BCPS, although these changes did not approach significance (PA in$\operatorname{dex}, p=0.21$; lower lobe index, $p=0.59$ ). There was no relationship between duration from BCPS to postBCPS angiography and changes in any PA index (Fig. 2), an observation that was independent of other covariates or factors, such as pre-BCPS PA indices and PA repair. There were significant inverse correlations between pre-BCPS total PA index and pre-BCPS to post-BCPS change in PA index $(R=-0.65$ [95\% CI: -0.87 to -0.44$], p<0.001)$ and between pre-BCPS lower lobe index and pre-BCPS to post-BCPS change in lower lobe index $(R=-0.60$ [95\% CI: -0.92 to $-0.29], p<0.001$ ), which are depicted in Fig. 3. These correlations were also significant individually for both the right and the left sides, with pre-BCPS right PA index $(R=0.74$ [95\% CI: -0.98 to -0.49$], p<0.001)$, left PA index ( $R=-0.61$ [95\% CI: -0.84 to -0.38 ], $p<0.001)$, right lower lobe index $(R=-0.77[95 \%$ CI: -0.99 to -0.56$], p<0.001$, and left lower lobe index $(\mathrm{R}=-0.36$ [95\% CI: -0.70 to -0.02 ], $p=0.026)$, correlating inversely with pre-BCPS to post-BCPS changes in right PA index, left PA index, right lower lobe index, and left lower lobe index, respectively.

Total PA index $(p=0.001)$, left PA index $(p=$ $0.02)$, and right $\mathrm{PA}$ index $(p=0.018)$ all increased significantly more from pre-BCPS to post-BCPS angiography in patients who underwent $\mathrm{PA}$ repair at the time of BCPS than in those who did not (Fig. 4). However, there was no correlation between PA repair at BCPS and changes in left, right, or total 


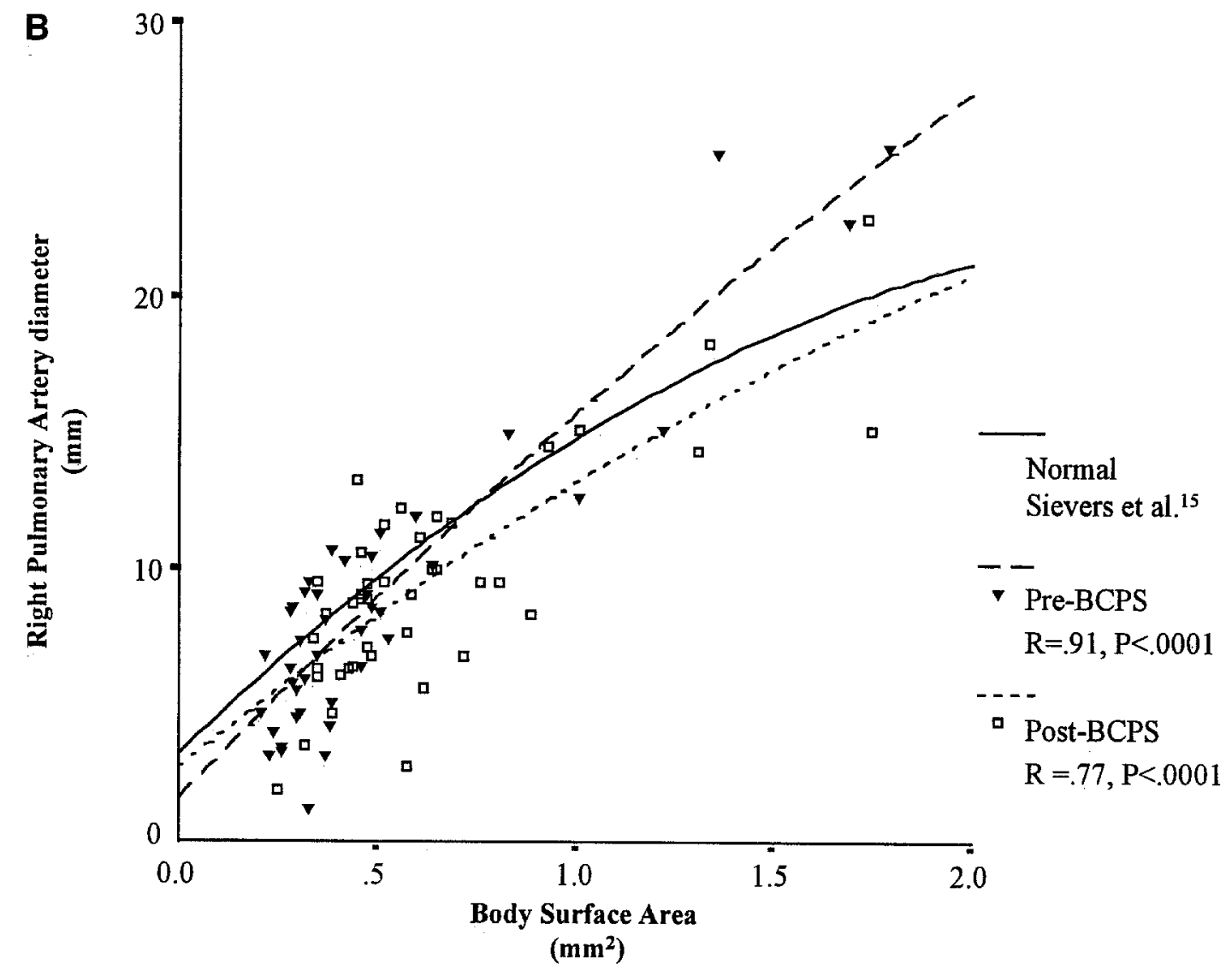

Fig. 1B. Pre-BCPS and post-BCPS right PA diameters (bifurcation) plotted against body surface area and compared with normal values published by Sievers and associates. ${ }^{15}$

Table III. Pre-BCPS and post-BCPS hemodynamic and oximetry data

\begin{tabular}{|c|c|c|c|}
\hline Variable & Pre-BCPS & Post-BCPS & $\begin{array}{c}p \\
\text { Value }\end{array}$ \\
\hline Body surface area $\left(\mathrm{mm}^{2}\right)$ & $0.56 \pm 0.42$ & $0.68 \pm 0.38$ & $<0.0001$ \\
\hline Arterial oxygen saturation $(\%)$ & $79.7 \pm 7.0$ & $82.0 \pm 6.3$ & 0.10 \\
\hline Mean PA pressure $(\mathrm{mm} \mathrm{Hg})$ & $14.7 \pm 7.5$ & $11.8 \pm 4.8$ & 0.01 \\
\hline Ventricular end-diastolic pressure $(\mathrm{mm} \mathrm{Hg})$ & $7.6 \pm 2.8$ & $5.8 \pm 3.3$ & 0.003 \\
\hline Pulmonary vascular resistance (Wood units) & $2.4 \pm 1.3$ & NA & \\
\hline Pulmonary/systemic blood flow ratio & $1.74 \pm 1.29$ & NA & \\
\hline Indexed pulmonary blood flow $\left(\mathrm{ml} / \mathrm{min} / \mathrm{m}^{2}\right)$ & $4.0 \pm 2.8$ & $\mathrm{NA}$ & \\
\hline Effective pulmonary blood flow $\left(\mathrm{ml} / \mathrm{min} / \mathrm{m}^{2}\right)$ & $1.5 \pm 0.6$ & NA & \\
\hline Pulmonary vascular compliance $\left(\mathrm{mm}^{2} / \mathrm{mm} \mathrm{Hg} / \mathrm{m}^{2}\right)$ & $54.2 \pm 31.3$ & NA & \\
\hline
\end{tabular}

Data are presented as mean \pm standard deviation. $N A$, Not available.

${ }^{*} p$ Values indicate significance of differences between pre-BCPS and post-BCPS values by paired two-tailed $t$ test.

lower lobe index (Fig. 4). As Fig. 4 also shows, patients with an extra source of pulmonary blood flow exhibited less decrease (though not to a statistically significant degree; $p=0.07)$ in lower lobe index than those with BCPS as the only source of pulmonary blood flow, regardless of whether they underwent PA repair. Extra pulmonary blood flow did not effect significant changes in PA index in patients with or without PA augmentation.

Total, left, and right lower lobe index did not differ significantly between patients with unilateral and bilateral BCPS, either before or after BCPS 
Table IV. Post-BCPS PA sizes and indices and change from pre-BCPS to post-BCPS

\begin{tabular}{|c|c|c|c|c|c|c|c|c|c|c|c|c|}
\hline & \multicolumn{5}{|c|}{ Right $P A$} & \multicolumn{5}{|c|}{ Left $P A$} & \multicolumn{2}{|c|}{ Total\$ } \\
\hline & $\begin{array}{c}\text { Origin } \\
\text { diameter* }\end{array}$ & $\begin{array}{c}N P \dagger \\
\text { diameter* }\end{array}$ & $\begin{array}{c}L L \\
\text { diameter* }\end{array}$ & $\begin{array}{l}\text { Origin } \\
\text { indext }\end{array}$ & $\begin{array}{c}L L \\
\text { indexf }\end{array}$ & $\begin{array}{c}\text { Origin } \\
\text { diameter }^{*}\end{array}$ & $\begin{array}{c}N P \dagger \\
\text { diameter* }\end{array}$ & $\begin{array}{c}L L \\
\text { diameter* }\end{array}$ & $\begin{array}{l}\text { Origin } \\
\text { indext }\end{array}$ & $\begin{array}{c}L L \\
\text { indext }\end{array}$ & $P A$ indext & $L L$ indert \\
\hline \multicolumn{13}{|l|}{$\begin{array}{c}\text { Post-BCPS } \\
\text { values }\end{array}$} \\
\hline All patients & $9.8 \pm 4.0$ & $8.6 \pm 3.9$ & $8.6 \pm 3.6$ & $124 \pm 62$ & $90 \pm 37$ & $9.4 \pm 4.1$ & $8.1 \pm 3.6$ & $8.3 \pm 3.4$ & $115 \pm 67$ & $91 \pm 53$ & $237 \pm 100$ & $177 \pm 70$ \\
\hline \multicolumn{13}{|l|}{$\begin{array}{l}\text { Additional } \\
\text { PBF }\end{array}$} \\
\hline Yes & $10.5 \pm 4.2$ & $9.4 \pm 4.2$ & $9.4 \pm 3.8$ & $130 \pm 58$ & $98 \pm 35$ & $10.3 \pm 4.1$ & $8.9 \pm 3.6$ & $9.2 \pm 3.6$ & $127 \pm 68$ & $104 \pm 57$ & $254 \pm 92$ & $196 \pm 68$ \\
\hline No & $8.3 \pm 3.2$ & $7.2 \pm 3.0$ & $7.0 \pm 2.4$ & $112 \pm 70$ & $75 \pm 37$ & $7.7 \pm 3.8$ & $6.5 \pm 3.1$ & $6.5 \pm 2.4$ & $93 \pm 59$ & $65 \pm 30$ & $205 \pm 110$ & $140 \pm 59$ \\
\hline \multicolumn{13}{|l|}{$\begin{array}{r}\text { Bilateral } \\
\text { BCPS }\end{array}$} \\
\hline Yes & $8.9 \pm 3.4$ & $7.6 \pm 3.2$ & $8.0 \pm 2.6$ & $126 \pm 59$ & $100 \pm 38$ & $8.9 \pm 4.0$ & $7.5 \pm 2.7$ & $8.1 \pm 3.2$ & $128 \pm 76$ & $103 \pm 51$ & $254 \pm 117$ & $203 \pm 79$ \\
\hline No & $10.1 \pm 4.2$ & $9.0 \pm 4.1$ & $8.8 \pm 3.9$ & $123 \pm 64$ & $86 \pm 36$ & $9.5 \pm 4.2$ & $8.3 \pm 3.8$ & $8.3 \pm 3.6$ & $110 \pm 64$ & $87 \pm 53$ & $230 \pm 94$ & $168 \pm 65$ \\
\hline \multicolumn{13}{|l|}{ PA repair } \\
\hline Yes & $10.8 \pm 3.4$ & $9.2 \pm 4.0$ & $8.8 \pm 2.9$ & $131 \pm 47$ & $89 \pm 30$ & $9.7 \pm 4.1$ & $7.8 \pm 3.0$ & $8.7 \pm 3.3$ & $112 \pm 69$ & $92 \pm 55$ & $240 \pm 99$ & $181 \pm 74$ \\
\hline No & $8.8 \pm 4.3$ & $8.1 \pm 3.9$ & $8.4 \pm 4.1$ & $118 \pm 73$ & $91 \pm 42$ & $9.1 \pm 4.2$ & $8.3 \pm 4.0$ & $7.9 \pm 3.6$ & $117 \pm 67$ & $90 \pm 51$ & $234 \pm 103$ & $174 \pm 68$ \\
\hline \multicolumn{13}{|l|}{$\begin{array}{l}\text { Pre-BCPS to } \\
\text { post- } \\
\text { BCPS } \\
\text { changes }\end{array}$} \\
\hline All patients & $1.3 \pm 4.0$ & & $0.8 \pm 2.7$ & $0.3 \pm 89$ & $-10 \pm 55$ & $0.4 \pm 4.2$ & & $1.0 \pm 2.6$ & $-18 \pm 73$ & $-6 \pm 49$ & $-14 \pm 128$ & $-23 \pm 102$ \\
\hline \multicolumn{13}{|l|}{$\begin{array}{c}\text { Additional } \\
\text { PBF }\end{array}$} \\
\hline Yes & $0.7 \pm 3.8$ & & $1.1 \pm 3.2$ & $-6 \pm 69$ & $1.5 \pm 58$ & $0.2 \pm 4.0$ & & $1.2 \pm 1.7$ & $-19 \pm 81$ & $-5 \pm 28$ & $-35 \pm 107$ & $-10 \pm 52$ \\
\hline No & $2.6 \pm 3.8$ & & $0.5 \pm 3.3$ & $13 \pm 71$ & $-32 \pm 60$ & $0.9 \pm 4.1$ & & $0.8 \pm 1.7$ & $-19 \pm 84$ & $-7 \pm 29$ & $22 \pm 110$ & $-49 \pm 54$ \\
\hline \multicolumn{13}{|l|}{ Bilateral } \\
\hline Yes & $0.7 \pm 2.1$ & & $1.5 \pm 2.6$ & $-17 \pm 57$ & $3 \pm 64$ & $1.2 \pm 1.7$ & & $1.8 \pm 2.2$ & $-4 \pm 50$ & $16 \pm 47$ & $-23 \pm 84$ & $16 \pm 83$ \\
\hline No & $1.5 \pm 4.5$ & & $0.7 \pm 2.7$ & $7 \pm 85$ & $-14 \pm 51$ & $0.1 \pm 4.8$ & & $0.8 \pm 2.8$ & $-25 \pm 80$ & $-15 \pm 47$ & $-14 \pm 130$ & $-40 \pm 80$ \\
\hline \multicolumn{13}{|l|}{ PA repair } \\
\hline Yes & $2.3 \pm 4.9$ & & $1.1 \pm 3.0$ & $29 \pm 83$ & $-8 \pm 60$ & $1.8 \pm 3.7$ & & $1.0 \pm 2.3$ & $8 \pm 63$ & $2 \pm 46$ & $40 \pm 113$ & $-13 \pm 72$ \\
\hline No & $0.2 \pm 2.6$ & & $0.7 \pm 2.3$ & $-30 \pm 61$ & $-10 \pm 51$ & $-0.8 \pm 4.3$ & & $1.2 \pm 2.9$ & $-42 \pm 74$ & $-13 \pm 51$ & $-70 \pm 94$ & $-32 \pm 96$ \\
\hline
\end{tabular}

Data are presented as mean \pm standard deviation. $B C P S$, Bidirectional cavopulmonary shunt; $L L$, lower lobe; $N P$, narrowest point; $P A$, pulmonary artery; $P B F$, pulmonary blood flow.

"Diameters are given in millimeters.

†NP diameter changes are not given, because the NP site differed from before to after BCPS in many patients.

$\ddagger$ Index units are square millimeters per square meter.

$\S$ Total refers to the sum total of left and right indices, averaged.

( $p>0.35$ in all cases). However, the difference in pre-BCPS to post-BCPS changes in left lower lobe index $(p=0.078)$ and total lower lobe index $(p=$ $0.064)$ between patients who underwent unilateral and bilateral BCPS approached significance. Changes in right PA diameters and indices among patients with bilateral BCPS showed no consistent pattern and did not approach significance. There was no significant pre-BCPS or post-BCPS difference in right versus left lower lobe index between patients with unilateral and bilateral BCPS $(p>$ 0.50 ). Similarly, there was no significant difference in right versus left lower lobe index changes from pre-BCPS to post-BCPS between patients with unilateral and bilateral BCPS $(p>0.50)$.
Twenty-two patients (47\%) were observed to have a total of 48 quantifiable systemic-PA collaterals at post-BCPS angiography, including 20 patients in whom no collaterals were noted on pre-BCPS angiograms. The presence or new development of arterial collaterals did not correlate with changes in PA diameters or indices.

Post-BCPS hemodynamic and oximetric data are summarized in Table II.

Fontan completion. Of the 47 patients included in the study group, 24 underwent a modified Fontan operation after post-BCPS catheterization, a median of 18.0 months after BCPS (range 1 to 47 months). There was one early death from a cerebrovascular accident after a modified Fontan operation 


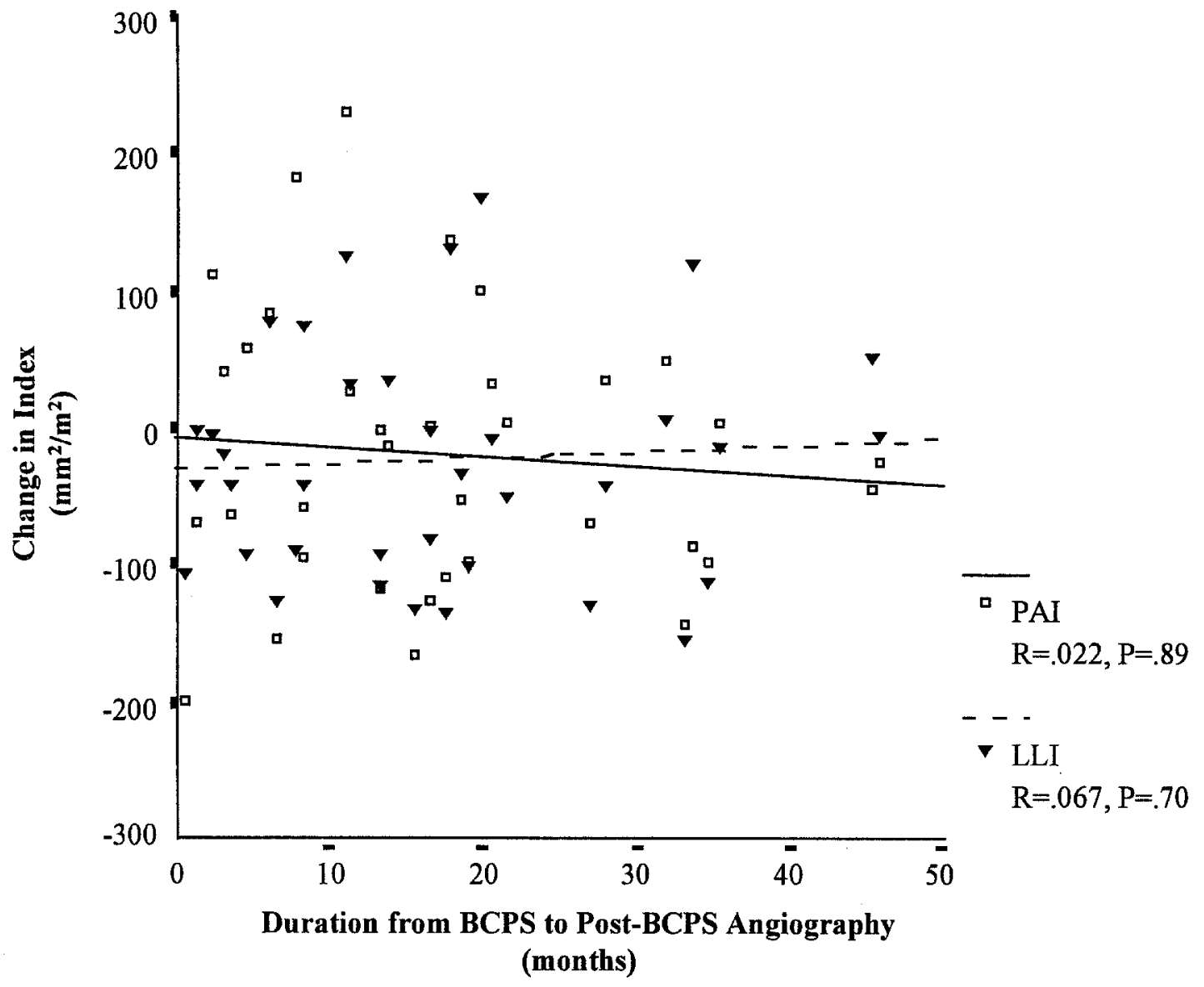

Fig. 2. Changes in total PA index $(P A I)$ and lower lobe index $(L L I)$ as a function of the duration of time between BCPS and post-BCPS angiography.

and repair of a stenotic pulmonary venous orifice (in a patient with a post-BCPS PA index of $305 \mathrm{~mm}^{2} /$ $\mathrm{m}^{2}$ ). Otherwise, there was no significant morbidity early after Fontan completion. The duration of chest tube drainage ranged from 3 to 53 days (median 9 days). Fontan pressures on the first postoperative day ranged from 7 to $16 \mathrm{~mm} \mathrm{Hg}$ (median $13 \mathrm{~mm} \mathrm{Hg}$ ).

PA index among patients having the Fontan procedure ranged from 96 to $431 \mathrm{~mm}^{2} / \mathrm{m}^{2}$ (median 252 $\mathrm{mm}^{2} / \mathrm{m}^{2}$ ). In general, trends in PA size changes and hemodynamic changes from pre-BCPS to postBCPS angiography were the same as in the overall cohort. None of the hemodynamic or angiographic factors analyzed from pre-BCPS to post-BCPS catheterization correlated with early outcomes after the Fontan operation, including mortality, Fontan pressure, and duration of chest tube drainage.

\section{Discussion}

Although reports evaluating the effect of PA size on outcomes of the modified Fontan operation have reached different conclusions, it is generally agreed that PA size can have an influence on the success of a Fontan procedure. ${ }^{1-6}$ Many of the patients studied in these reports underwent a Fontan operation before the widespread use of BCPS for pre-Fontan palliation. Because pulmonary blood flow, which is widely believed to stimulate PA growth, is decreased in the setting of BCPS, there is concern that use of BCPS for palliation in patients with single ventricle may have adverse implications for outcomes after subsequent completion Fontan. Previous reports of PA growth after BCPS describe either an increase or no change in PA diameter ${ }^{13,14}$ or a decrease in the diamater of the PA contralateral to the cavopulmonary anastomosis. ${ }^{12}$ However, all of these groups 


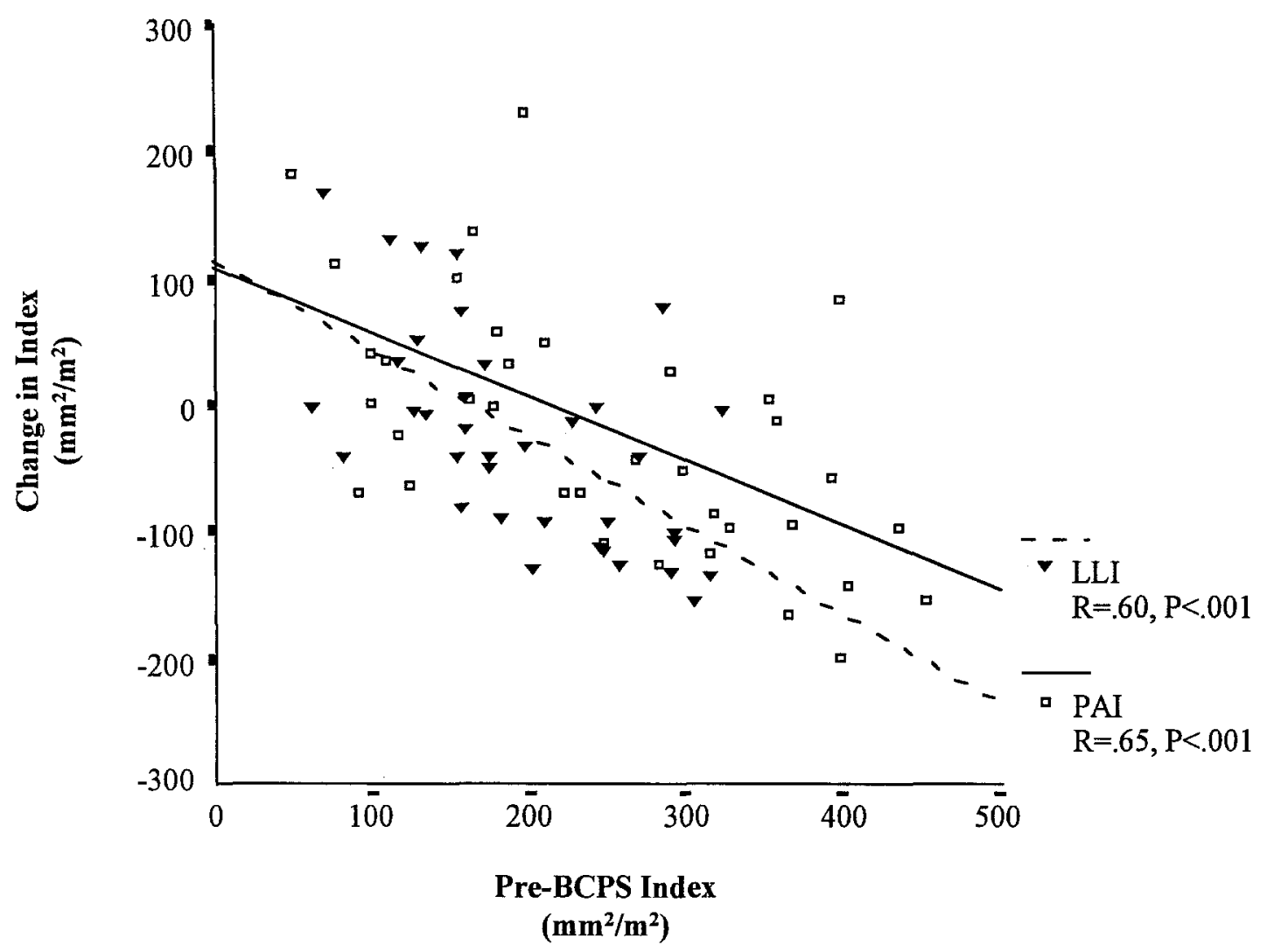

Fig. 3. Changes in total PA index $(P A I)$ and lower lobe index $(L L I)$ as a function of pre-BCPS PAI and LLI, respectively.

found that PA sizes, assessed as either Nakata indices or $\mathrm{Z}$ scores, were relatively smaller after BCPS, which they postulated to represent decreased growth. In the two groups that measured PA size in terms of the Nakata index, the mean post-BCPS PA index was found to be significantly smaller than the pre-BCPS index,${ }^{12,13}$ whereas the group that calculated $\mathrm{Z}$ scores observed a statistically insignificant decrease after BCPS. ${ }^{14}$ In the present study, we found that changes in PA size varied considerably. The absolute diameters of the right and left lower lobe PAs increased significantly, but the calculated indices did not change significantly although there was a tendency to decrease.

The effect of BCPS on PA growth may have implications for the timing of both BCPS and subsequent Fontan operation. Mendelsohn and associates $^{12}$ reported that patients with BCPS for longer than 15 months had significantly greater decreases in PA size than those with BCPS in place for 15 months or less. However, the statistical analysis they presented to prove the validity of this observation treated time after BCPS as a dichotomous variable, rather than a continuous one. The analysis involved a simple comparison of means, performed separately for patients with BCPS for longer than and shorter than 15 months. Analysis of variance or other methods that would have accounted for different baseline values and relative degrees of change in PA size were not performed. In contrast to these findings, linear regression analysis performed on the present series of patients showed absolutely no correlation between changes in PA index or lower lobe index and duration from BCPS (or from preBCPS angiography) to post-BCPS angiographic study (Fig. 2).

It is difficult to account for all of the factors that contribute to changes in angiographically measured PA size. Although PA pressure and pulmonary blood flow certainly contribute to PA distention ${ }^{16,17}$ and are likely to affect growth, neither of these factors appeared to correlate with changes in PA size after BCPS, either alone or when analyzed as covariates with PA size or with other factors. An 


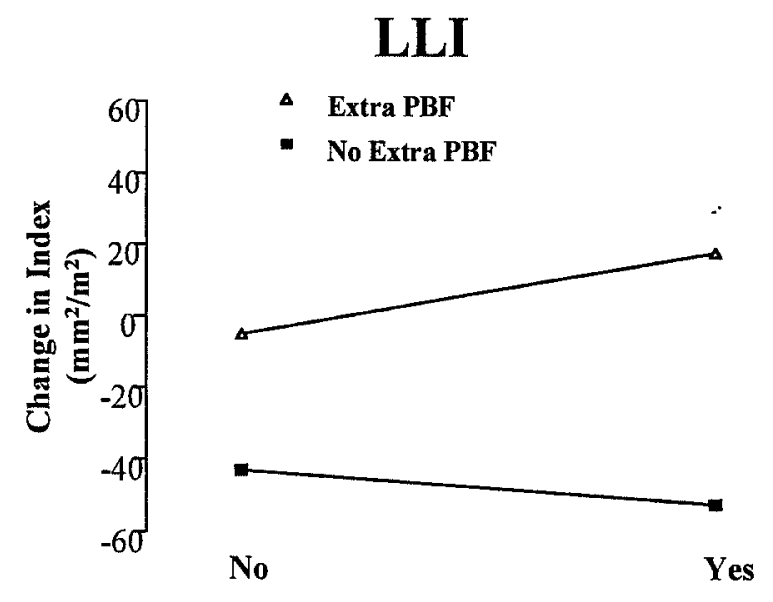

A. Pulmonary Artery Plasty at BCPS

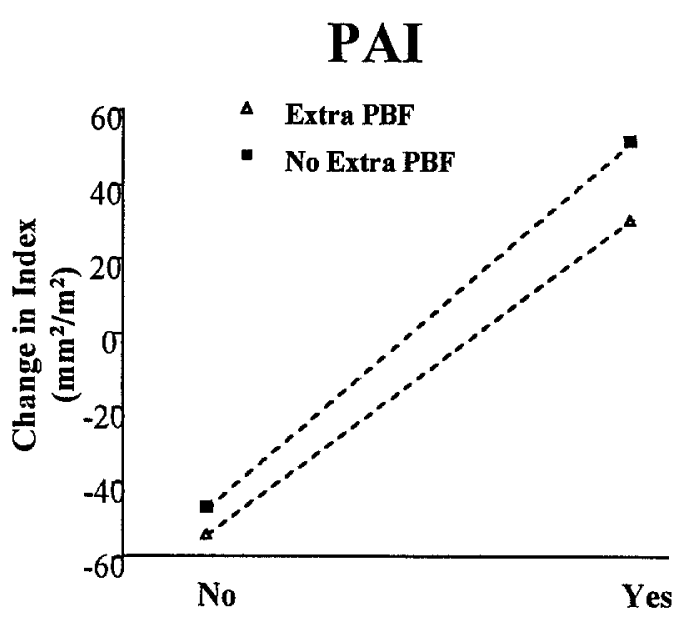

B. Pulmonary Artery Plasty at BCPS

Fig. 4. Profile plot of changes in lower lobe index (LLI) (A) and total PA index (PAI) (B) according to whether PA augmentation procedures were or were not performed at BCPS and whether a source of pulmonary blood flow $(P B F)$ in addition to the BCPS was present.

important consideration in analyzing PA size in patients who have undergone BCPS, as well as those who have undergone previous systemic-PA shunts, is the role of surgical distortion. Although we found very little change in either PA index or lower lobe index after BCPS in the whole group, we found that right PA index, left PA index, and total PA index were affected significantly by $P A$ repair at the time of BCPS, with significantly less decrease (or greater increase) in these measures among patients who had undergone PA repair than those who had not. Importantly, none of the previous reports of PA growth after BCPS analyzed the effect of PA repair on changes in PA size in their patients, and none of them looked specifically at size changes in the lower lobe arteries, which are generally not involved in PA augmentation procedures. ${ }^{12-14}$ Mendelsohn and colleagues ${ }^{12}$ mention that two of 30 patients required patch augmentation at the time of BCPS and seven patients required intervention for PA hypoplasia or stenosis (the time of this intervention is not stated clearly, although the context of the statement suggests that it was at BCPS). Slavik and coworkers ${ }^{14}$ mention that seven of 39 patients underwent central PA augmentation at the time of BCPS. Neither of these groups described any specific analysis that takes these issues into consideration.

Because the branch PAs are often distorted by previous systemic-PA shunts in patients undergoing BCPS, because the cavopulmonary anastomosis almost invariably extends to the takeoff of the upper lobe branch, and because some change in PA structure inevitably follows the BCPS operation, regardless of whether PA augmentation procedures are performed, it is our opinion that measurement of the central PAs may represent a dubious approach to the analysis of PA growth in the context of BCPS. Because of the aforementioned considerations, we believe that measurement of the lower lobe arteries involves fewer confounding factors and is therefore a more appropriate approach to assessing PA size changes in this group of patients.

Two additional factors that may affect either the PA index or lower lobe index are the presence of bilateral BCPS and/or an additional source of pulmonary blood flow. It has been reported that the PA contralateral to a unilateral BCPS (usually left) decreased significantly in both diameter and index after BCPS, whereas the ipsilateral PA did not. ${ }^{12}$ We observed that total and left lower lobe index decreased less among patients who underwent bilateral BCPS than among those in whom the BCPS was unilateral, and these differences approached significance. The practical relevance of these findings may be minimal, serving only to support previous observations that pulmonary blood flow is more efficiently distributed in the presence of bilateral BCPS and to recommend the performance of bilateral BCPS in the rare cases in which two sizable venae cavae and a substantial bridging vein are present. Another group found that patients without an extra source of pulmonary blood flow exhibited relatively less left 
PA growth. ${ }^{14}$ In our series, extra pulmonary blood flow did not have a significant effect on changes in PA index or lower lobe index, although there was a trend $(p=0.07)$ toward less decrease in total lower lobe index in patients with an additional source of pulmonary flow. Though none of the reported studies, including the present report, demonstrate a clear PA growth benefit of maintaining extra pulmonary blood flow, our finding that the lower lobe index decreases to a relatively lesser degree in the presence of extra pulmonary blood flow may be an important consideration in the ongoing dialog surrounding the benefits and drawbacks of extra pulmonary blood flow with BCPS.

Although size changes are almost certainly due to a multiplicity of factors, including growth, we found a significant inverse correlation between pre-BCPS PA indices (PA index and lower lobe index) and change in these indices from pre-BCPS to postBCPS angiography. This observation is consistent with previous reports of PA growth in patients who have undergone a variety of procedures to augment pulmonary blood flow, including BCPS, ${ }^{12}$ BlalockTaussig shunt, ${ }^{18}$ Waterston anatomosis, ${ }^{19}$ and assorted palliative procedures for tetralogy of Fallot. ${ }^{20}$

Of the 47 patients in the present series, 24 have undergone completion Fontan, between 1 and 47 months after BCPS. The median PA index in these patients was $252 \mathrm{~mm}^{2} / \mathrm{m}^{2}$, and five patients had a PA index of less than $150 \mathrm{~mm}^{2} / \mathrm{m}^{2}$. The one death after a Fontan operation occurred in a patient with a PA index of $305 \mathrm{~mm}^{2} / \mathrm{m}^{2}$. These results confirm previous reports that have shown that a successful Fontan operation can be carried out regularly in patients with a PA index under $150 \mathrm{~mm}^{2} / \mathrm{m}^{2} .2,5,6,12$ However, we continue to perform PA repair in the majority of our patients, at the time of Fontan operation, because we believe that the PA index is not an adequate measure for assessing the central PAs, an argument which is supported by the findings of this study and which we have also made with respect to patients undergoing repair of tetralogy of Fallot. ${ }^{21}$ Instead, the lower lobe index may be a more appropriate measure of PA size and growth and a better means of assessing the relationship between PA size and Fontan outcomes.

The primary shortcoming of the present study is that immediate post-BCPS angiographic data were not available, which is also the case with the three other published reports on this topic. ${ }^{12-14}$ The baseline from which PA size changes were measured, namely, pre-BCPS angiographic measurements, probably does not reflect early post-BCPS PA size in the majority of cases. For example, the patient in whom the greatest decrease in total PAI was observed in this study underwent post-BCPS angiography only 1 month after BCPS, before conversion to a modified Fontan that was performed because of persistent low saturations after BCPS. Thus it is difficult to make any determination about growth on the basis of these results. Nevertheless, it appears from our experience and analysis that the changes that occur in the size of the PAs after BCPS are not clinically significant, at least in the short and medium term, or if a Fontan procedure is performed within 2 to 3 years. However, this study does not address the physiologic effects of PA changes in the setting of BCPS maintained as a long-term palliation or when conversion to a Fontan physiology is performed after a longer period of BCPS.

\section{REFERENCES}

1. Girod DA, Rice MJ, Mair DD, Julsrud PR, Puga FJ, Danielson GK. Relationship of pulmonary artery size to mortality in patients undergoing the Fontan operation. Circulation 1985;72(Suppl):II93-6.

2. Bridges ND, Farell PE, Pigott JD, Norwood WI, Chin AJ. Pulmonary artery index: a nonpredictor of operative survival in patients undergoing modified Fontan repair. Circulation 1989;80(Suppl):I216-21.

3. Nakata S, Imai Y, Takanashi Y, Kurosawa H, Tezuka K, Nakazawa M. A new method for quantitative standardization of cross-sectional areas of the pulmonary arteries in congenital heart diseases with decreased pulmonary blood flow. J Thorac Cardiovasc Surg 1984;88:610-9.

4. Fontan F, Fernandez, Costa F, et al. The size of the pulmonary arteries and the results of the Fontan operation. J Thorac Cardiovase Surg 1989;98:711-24.

5. Knott-Craig CJ, Julsrud PR, Schaff HV, Puga FJ, Danielson GK. Pulmonary artery size and clinical outcome after the modified Fontan operation. Ann Thorac Surg 1993;55:64651.

6. Senzaki H, Isoda T, Ishizawa A, Hishi T. Reconsideration of criteria for the Fontan operation: influence of pulmonary artery size on postoperative hemodynamics of the Fontan operation. Circulation 1994;89:266-71.

7. Bridges ND, Jonas RA, Mayer JE, Flanagan MF, Keane JF, Castaneda AR. Bidirectional cavopulmonary anastomosis as interim palliation for high-risk Fontan candidates: early results. Circulation 1990;82(Suppl):IV170-6.

8. Pridjian AK, Mendelsohn AK, Lupinetti FM, et al. Usefulness of the bidirectional Glenn procedure as staged reconstruction for the functional single ventricle. Am J Cardiol 1993;71:959-62.

9. Reddy VM, McElhinney DB, Moore P, Hanley FL. Outcomes after bidirectional cavopulmonary shunt in infants under six months of age [abstract]. J Am Coll Cardiol 1996;27:370A.

10. Chang AC, Hanley FL, Wernovsky G, et al. Early bidirec- 
tional cavopulmonary shunt in young infants: postoperative course and early results. Circulation 1993;88(Pt 2):149-58.

11. Jarmakani JMM, Graham TP, Benson DW, Canent RV, Greenfield JC. In vivo pressure-radius relationships of the pulmonary artery in children with congenital heart disease. Circulation 1971;43:585-92.

12. Mendelsohn AM, Bove EL, Lupinetti FM, Crowley DC, Lloyd TR, Beekman RH. Central pulmonary artery growth patterns after the bidirectional Glenn procedure. J Thorac Cardiovasc Surg 1994;107:1284-90.

13. Penny DJ, Pawade A, Wilkinson JL, Karl TR. Pulmonary artery size after bidirectional cavopulmonary connection. J Card Surg 1995;10:21-6.

14. Slavik Z, Webber SA, Lamb RK, et al. Influence of bidirectional superior cavopulmonary anastomosis on pulmonary artery growth. Am J Cardiol 1995;76:1085-7.

15. Sievers HH, Onnasch DGW, Lange PE, Bernhard A, Heintzen PH. Dimensions of the great arteries, semilunar valve roots, "and right ventricular outflow tract during growth: normative angiocardiographic data. Pediatr Cardiol 1983;4: 189-96.

16. Harris P, Heath D, Apostopoulos A. Extensibility of the pulmonary trunk in heart disease. Br Heart J 1965;27:660-6.

17. Pasierski TJ, Starling RC, Binkley PF, Pearson AC. Echocardiographic evaluation of pulmonary artery distensibility. Chest 1993;103:1080-3.

18. Gale AW, Arciniegas E, Green EW, Blackstone EH, Kirklin JW. Growth of the pulmonary anulus and pulmonary arteries after the Blalock-Taussig shunt. J Thorac Cardiovasc Surg 1979;77:459-65.

19. Alfieri O, Blackston EH, Parenzan L. Growth of the pulmonary anulus and pulmonary arteries after the Waterston anastomosis. J Thorac Cardiovasc Surg 1979;78:440-4.

20. Laas J, Engeser U, Meisner H, et al. Tetralogy of Fallot: development of hypoplastic pulmonary arteries after palliation. Thorac Cardiovasc Surg 1984;32:133-8.

21. Reddy VM, Liddicoat JR, McElhinney DB, Brook MM, Stanger P, Hanley FL. Routine primary repair of tetralogy of Fallot in neonates and infants under three months of age. Ann Thorac Surg 1995;60:S592-6.

\section{Discussion}

Dr. Thomas R. Karl (Melbourne, Australia). PA size is important for Fontan outcome, both in the short term and in the long term. I base this statement on studies of 300 Fontan operations performed in our own institution, and I think that this conclusion is supported by many, although not all, publications in the world literature.

Part of the problem is that much of this information was generated in the era of the one-stage Fontan, that is, an operation performed without preliminary BCPS. This shunt is important because it gives us a window through which we can prospectively view post-Fontan hemodynamics. Patients who have a persistently elevated central venous pressure or poor atrioventricular valve or ventricular function after a BCPS are unlikely to be good Fontan candidates irrespective of PA size. Conversely, patients who have small PAs but have excellent hemodynamics after the BCPS are likely to have good results after a Fontan operation. Because of this selection process, it will be difficult to say that PA size per se has a strong effect on Fontan outcome in the current era.
We agree that the use of a lower lobe index is probably a better way to measure the PA tree than the Nakata index because, after all, our concern is a generalized hypoplasia rather than a discrete proximal stenosis that can be treated with a surgical procedure. However, the use of the Nakata index will underestimate the problems associated with the small PA, not overestimate them. Use of the Nakata index alone rather than the lower lobe index could not explain the difference in your findings versus those of other series in which PAs did not develop well after the BCPS.

We have looked at a remarkably similar cohort of patients in Melbourne, and we did see a significant decrease in Nakata index that was not related to the change in pulmonary/systemic flow ratio or to the interval from BCPS to postoperative catheter follow-up. This experience led us to conclude that the BCPS is a suboptimal stimulus for PA growth and should not be used solely for that indication, although, of course, there are many other good indications.

The only difference that I can see between your series and the Melbourne series is that patients in San Francisco were much more likely to have a second source of pulmonary blood flow added or preserved at the time of BCPS. In the Melbourne series, the BCPS was the unique source for every patient.

I would ask the following questions:

One, what were the selection criteria used to decide about use of an alternate source of pulmonary blood flow?

Two, assuming some selection process was applied, could it have obscured differences that might have been seen in PA growth after BCPS (as compared with a randomization into alternate source versus no alternate source)?

Three, do you believe that patients who are otherwise in suitable condition should have a Fontan operation irrespective of PA size, or would you impose some selection criteria here as well?

Finally, should we not qualify the statement that PA size does not have an effect on Fontan outcome, given the fact that there probably has been some selection process and this hypothesis has not really been tested at the lower end of the PA size spectrum, especially in patients who have a one-stage procedure?

Dr. Reddy. Thank you, Dr. Karl, for your comments. Our present selection criteria for an extra source of pulmonary blood flow are very subjective. If the patient has an extra source of antegrade blood flow, we generally tend to decrease it after measuring the pressures after completion of the BCPS. We put in a PA catheter and try to reduce the pressures to $15 \mathrm{~mm} \mathrm{Hg}$ or less. At that point we allow any extra source of blood flow to remain.

If the patient does not have antegrade blood flow and if the BCPS is placed at the site of a previous shunt we take down the shunt and construct a BCPS. Those patients do not receive any extra source of pulmonary blood flow unless their saturations are very low after the BCPS. If the shunt enters the PA at a site other than where the BCPS is going to go, then we generally try to leave it alone so that we can perform the BCPS without cardiopulmonary bypass and try to reduce its size again by measuring the PA pressures. That is our current approach. We have 
neither intentionally left an extra source of blood flow nor added one in our patients. However, if there is a source of blood flow that allows us to do the BCPS without the need for bypass, we generally leave it alone and try to reduce it after we finish the shunt.

With regard to the effect on $\mathrm{PA}$ growth and the extra source of pulmonary blood flow, the change in the lower lobe diameters and index approaches significance in patients who have an extra source of pulmonary blood flow, but it does not reach significance. However, on the basis of the physiology and effect of flow on growth, I would presume that if we wait longer the extra source of pulmonary blood flow would allow better growth.

Finally, the literature contains conflicting information regarding the effect of $\mathrm{PA}$ size on the Fontan outcome. What we do not take into account when we measure the sizes is how flow is going to change the PAs. When we do a Fontan from a Glenn, we increase the pulmonary/systemic flow ratio from 0.5 to 1 . Thus, whether the actual change in size after the Fontan is a function of distensibility or not, if you perceive the tubes as being rigid and if their index is only 69, then maybe they are not suitable for the Fontan operation. However, they are not rigid tubes. When the pulmonary blood flow is increased, they may distend, and the distensibility is one factor that we cannot account for. Thus it would seem inappropriate to use the absolute sizes or the indices as a basis for selecting a cutoff point. We would rather look at the hemodynamics of the patient as a whole and at the pulmonary vascular resistance and then do a Fontan operation.

Also, just evaluating the PA index or the Nakata index is meaningless. The PAs can be surgically enlarged all the way into the lower lobe arteries also, so the Nakata index is irrelevant with regard to the Fontan procedure or any other procedure. More important is the distal vascular bed, and that cannot be assessed except by measuring pulmonary vascular resistance. I think the central PAs are inaccurate and probably should not be used at all in coming to any sort of conclusion. More objective criteria should be developed to assess the distal pulmonary vascular bed.

Dr. Karl. Do we really know that Fontan suitability does not depend on PA size whether analyzed as central measurements such as the Nakata index or a lower lobe index? Have we really tested that? I think that at this point that is an overstatement.

Dr. Reddy. Judging from our experience both in patients having the Fontan operation and in those with tetralogy of Fallot, I think central PAs are inaccurate and probably should not be used as a measure for outcome for Fontan procedures.

Dr. Richard A. Jonas (Boston, Mass.). You have measured the PA diameter before the bidirectional Glenn shunt and not immediately after the Glenn shunt. Thus it is indeed possible that there was a substantial reduction in diameter when you took away, perhaps, a high-pressure arterial shunt, and it is possible that there was significant growth from a point immediately after the Glenn until the time that you did the pre-Fontan angiogram. In other words, you may not be assessing the growth of the PAs in a fair way.

Dr. Reddy. I agree completely with you, Dr. Jonas. We have to be cautious in interpreting any of these changes as growth, because that is an interpretive assessment. That fact that the PA is distended by flow certainly does not mean growth. Similarly, if the flow is decreased, the PA can collapse and only the diameter in the collapsed state will be measured. Growth is sort of a fallacious term.

We do not have any angiograms taken immediately after a Glenn shunt. The earliest is 1 month after BCPS. It is very likely, I would say, that the artery size decreases immediately after the Glenn operation when the arterial shunt is taken off.

\section{Appendix}

Variables analyzed by linear regression, logistic regression, general factorial analysis of variance, and paired two-tailed $\mathrm{t}$ test

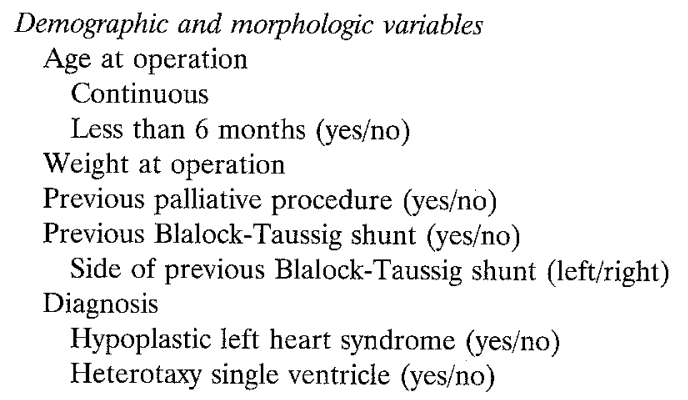

Catheterization and angiographic variables (pre-BCPS and post$B C P S$ absolute values, as well as absolute and percent changes from before to after BCPS, were considered as separate variables) Arterial oxygen saturation

Mean systemic arterial blood pressure

Mean PA blood pressure

Ventricular (dominant/single) end-diastolic pressure

Indexed pulmonary blood flow

Pulmonary/systemic blood flow ratio Continuous

Greater than 1.5 (yes/no)

Pulmonary vascular resistance

PA diameter

Right (bifurcation, lower lobe [LL], narrowest point [NP]) Left (bifurcation, LL, NP)

Pulmonary artery index

Total (bifurcation, LL, NP, NP as a percentage of bifurcation)

Right (bifurcation, LL, NP, NP as a percentage of bifurcation)

Left (bifurcation, LL, NP, NP as a percentage of bifurcation)

Percent difference between branch PA NP and bifurcation diameters

Continuous (left, right)

Less than $75 \%$ (significant branch PA stenosis)

Systemic-pulmonary collateral arteries

To left lung, right lung, either lung (yes/no)

Absent upper lobe blood flow, left or right (yes/no)

Operative variables

Bilateral BCPS (yes/no)

$P A$ augmentation (yes/no)

Intracardiac procedures performed (yes/no)

Extra source of pulmonary blood flow (yes/no) 
Type (shunt/antegrade)

Cardiopulmonary bypass (yes/no)

Time

Early postoperative variables

Death (yes/no)

BCPS takedown (yes/no)

Pulmonary blood flow-related reoperation (yes/no)

Duration of ventilator dependence

Effusions (chest tube drainage) greater than 7 days (yes/no)

Duration of hospital stay

Late postoperative variables

Pulmonary blood flow-related reoperation before Fontan

(yes/no)
Time between pre-BCPS and post-BCPS catheterization

Time between BCPS operation and post-BCPS catheterization

Variables relating to Fontan completion

Time between BCPS and Fontan completion

Early post-Fontan death (yes/no)

PA augmentation (yesino)

Duration of post-Fontan effusions (chest tube drainage)

Continuous; longer than 7 days (yes/no)

Duration of post-Fontan hospital stay

\section{ON THE MOVE?}

Send us your new address at least six weeks ahead

Don't miss a single issue of the journal! To ensure prompt service when you change your address, please photocopy and complete the form below.

Please send your change of address notification at least six weeks before your move to ensure continued service. We regret we cannot guarantee replacement of issues missed due to late notification.

\section{JOURNAL TITLE:}

Fill in the title of the journal here.

\section{OLD ADDRESS:}

Affix the address label from a recent issue of the journal here.

\section{NEW ADDRESS:}

Clearly print your new address here.

Name

Address

City/State/ZIP
COPY AND MAIL THIS FORM TO:

Journal Subscription Services

Mosby-Year Book, Inc.

11830 Westline Industrial Dr.

St. Louis, MO 63146-3318
OR FAX TO:

314-432-1158

N/Mosby
OR PHONE:

1-800-453-4351

Outside the U.S., call

314-453-4351 